\title{
An Efficient Traffic-Load and Link-Interference Aware Routing Metric for Multi Radio Multi Channel Wireless Mesh Networks Based on Link's Effective Capacity Estimation
}

\author{
Maheen Islam¹, M. Lutfar Rahman ${ }^{2} \&$ Mamun-Or-Rashid ${ }^{3}$ \\ ${ }^{1}$ Department of Computer Science and Engineering, East West University, Dhaka, Bangladesh \\ ${ }^{2}$ Daffodil International University, Dhaka, Bangladesh \\ ${ }^{3}$ Department of Computer Science and Engineering, University of Dhaka, Dhaka, Bangladesh \\ Correspondence: Maheen Islam, Department of Computer Science and Engineering, East West University, \\ Dhaka, Bangladesh. E-mail: maheen@ewubd.edu
}

Received: October 11, 2014

Accepted: October 16, $2014 \quad$ Online Published: October 30, 2014

doi: $10.5539 /$ cis.v7n4p129

URL: http://dx.doi.org/10.5539/cis.v7n4p129

\begin{abstract}
Routing metrics proposed for Wireless Mesh Networks (WMNs) has various concerns like hop count, packet transmission delay, power consumption, congestion control, load balance and message collision. The routing metric of expected effective capacity (EEC) proposed in this paper guarantees to a select a path providing maximum throughput and minimum delay. A forwarding link constituting routing path is characterized by its quality, capacity, traffic demand and the degree of intervention experienced due to inter-flow and intra-flow interference. Thus the bandwidth actually attainable on a link for a flow is affected by those link properties. Our proposed metric computes the attainable bandwidth for a flow over a path which actually reflects congestion, node delay and traffic pressure on the desired path. Experiments conducted on ns-2 simulations demonstrate that our proposed routing metric can achieve significant improvements in overall network throughput, minimize end-to-end delay and able to distribute network load.
\end{abstract}

Keywords: wireless mesh networks, routing metric, interference domain, congestion degree, link load

\section{Introduction}

Due to its potential performance, multihop Wireless Mesh Networks (WMN) has stirred much interest in both academia and commercial sectors (Akyildiz \& Wang, 2005) in recent years. The key reason that hinders the capacity of WMN is wireless interference. Employment of Multi Radio Multi Channel (MRMC) WMN architecture, in which each mesh router is equipped with multiple radios, which can configured to orthogonal frequency channels to allow simultaneous transmission among neighboring nodes, can alleviate the performance degradation. Hence, MRMC WMN can achieve higher system throughput than the traditional Single Radio Single Channel (SRSC) mesh network.

In recent years, many researchers paid hard endeavor to build routing metrics for MRMC WMN that achieve as high throughput as possible over the network. To reduce network-wide channel contentions, most of the existing works propose interference-aware or load-balancing routing metrics to select a path. Design of efficient routing schemes for an MRMC wireless mesh network is much more challenging compared to the SRSC case. Routing is dependent on channel assignment and vice versa. Wireless nodes operating over the same channel in a neighborhood have to share the channel capacity, and hence the amount of bandwidth assigned to a link depends on degree of congestion of sharing links in the neighborhood. Thus, channel assignment to radios affects the amount of bandwidth available on links, and hence the channel assignment problem must be jointly studied with the routing problem. However, the joint channel assignment and routing problem has been shown to be NP-complete (Alicherry \& Bhatia, 2006). Therefore, the proposals that recently appeared in the literature addressing such joint problem solve the channel assignment problem and the routing problem separately. Channels can be allocated to radios in two different approaches: traffic-independent and traffic-aware. In traffic-independent approach, routing and channel assignment function is carried separately with routing dependent on the result of channel assignment and is focused to maximize network connectivity and minimize interference without explicitly considering network load. Traffic-aware channel assignment, on the other hand, is 
affected by the traffic condition in the network. Traffic-aware channel assignment can be done separately but iteratively with routing. Both strategies have their advantages and disadvantages. Traffic-independent approach does not require interfaces to switch channels, and thus have lower overhead. However, they depend on the stable and predictable traffic patterns in the network. Traffic-aware strategies are involved in frequent channel switching, and thus have higher overhead than static strategies. In view of the above discussion, we adopt the traffic-independent approach in this work.

The routing metric of expected effective capacity (EEC) proposed in this paper selects a path consisting of maximum capacity links that can deliver a packet with the shortest end-to-end delay. The capacity of each link on a path is determined by traffic load and neighboring interference. In order to find the load on a link for a flow, each node considers queuing delay, backoff delay and transmission delay to estimate the packet service time; and monitors the buffer to estimate the level of queue utilization. In addition the end-to-end throughput over a multi-hop wireless network is particularly impacted by the interference among different hops, which can be classified into inter-flow and intra-flow interference. Due to concurrent transmission, links residing on the same interference domain is restricted to share the available network bandwidth among them. So considering the number of flows interfering to each other, we opt to find the actual allotted bandwidth to each link and incorporate it to compute EEC. The EEC routing metric also attempts to balance load in the network. A path with maximum value of EEC consists of the lightly loaded links with greater capacity and less interference, and thus balances the traffic from congested links.

The rest of the paper is organized as follows. Section 2 discusses the related work in the literature. Section 3 describes the system architecture and Section 4 presents the proposed protocol. Performance evaluation is carried out in Section 5 and the paper concludes in Section 6.

\section{Related Works}

MRMC WMN has been deployed to improve the performance of SRSC WMN. Routing and channel assignment (CA) play vital role to fully captivate the capacity of MRMC WMN. There are a number of centralized and distributed solutions for CA and routing in an MRMC network. JRCAR (Gálvez, \& Juan, 2013) routes and balances traffic at the flow level and adapts CA to real-time variations in traffic without prior knowledge of traffic. (Ramachandran \& Sheriff, 2008) propose centralized traffic-independent channel selection algorithm (TIC) to compute routes between the gateway and mesh routers and allocates channels to links on these routes. (Gardellin \& Das, 2011) and (Mohsenian-Rad \& Wong, 2007) proposes ILP formulations to calculate topology, channel allocation and routing, assuming prior knowledge of traffic matrix. (Dhananjay \& Zhang, 2009) propose a distributed load-independent $\mathrm{CA}$ and routing protocol, where different channels are assigned to links on a route from a node to specified gateway. CRAFT (Hong \& Warner, 2012) jointly optimizes routing and channel assignment by using an objective function that tries to maximize traffic transfer under given reliability of a link. In (Ding \& Yong, 2013), a dynamic channel allocation protocol (ADCA) assigns least congested channels to dynamic interfaces in the neighborhood and Interference and Congestion Aware Routing protocol (ICAR) defines a cost metric of each link based on link's flow rate, ETX (Cout \& Aguayo, 2003), load and interference probability. (Li \& Wu, 2012) propose a threshold-based control system called balanced control system (BCS), where traffic load and interference level is monitored to fix up a dynamic channel switching threshold value.

There are several previously proposed link quality based routing metrics. The ETX(Cout \& Aguayo, 2003) metric computes the expected number of transmissions (including retransmissions) needed to send a packet over a link, by measuring the forward and reverse packet delivery ratios (PDR) between a pair of neighboring nodes where a single radio channel is used. The ETT metric was proposed in (Draves \& Padhye, 2004) on a given link is defined as the expected time to send a 1500-byte packet at the rate that yields the highest throughput on that link. The ETOP metric proposed in (Jakllari \& Eidenbenz, 2012) accounts for the finite number of retransmission attempts at the link layer. A good routing metric should find paths with component links that have low loss ratio, high data rate and experience low levels of interference. Several research proposals include varying interference experienced by a link into the routing metric to find good quality paths. Authors in (Draves $\&$ Padhye, 2004), propose a new path metric, called weighted cumulative expected transmission time (WCETT), which explicitly accounts for the interference among links using the same channel. ETM (Kim \& Jakllari, 2011) routing metric captures number of links, link rates, success probability and link position along routing path. Considering packet drops closer to the destination are expensive in terms of retransmissions costs, ETM-based rate adaptation algorithm tunes the rate dynamically that ensures less aggressive rate increase on these links. The metric of interference and channel switching (MIC) (Yang \& Wang, 2005) incorporates both interflow and intra-flow interference. iAWARE (Subramanian \& Buddhikot, 2006) uses ETT weighted with interference ratio, which is ratio of SINR and SNR at receiver node, that takes into account the interference experienced by the link 
from its neighbors.

Works on (Li \& Cheng, 2008) and (Li, \& Cheng, 2013)focus on computing the achievable bandwidth when both inter- and intra-flow interferences are present. Expected end-to-end delay (EED) proposed in ( $\mathrm{Li}, \&$ Cheng, 2013) monitors the transmission failure probability to estimate the transmission delay, and counts the number of packets waiting in the buffer to estimate the queuing delay. EED is then integrated with a path metric called multiradio achievable bandwidth (MRAB) to accurately capture the impact of inter and intra-flow interference along a path. (Islam \& Alam, 2010) proposes a new routing metric EFT(Expected Forwarding time) for HWMP(IEEE 802.11s Task Group, 2008), where a forwarding node needs to estimate the number of retransmission and transmission rate, expected duration of idle slots during backoff, neighbor density and loads, transmission rate and packet size of neighbors, defer time due to contending high priority traffic and queuing delay.

To cope with unreliable and unpredictable wireless links, researchers (Biswas \& Morris, 2005), (Yuan \& Yang, 2005), (Rozner \& Seshadri, 2009), developed opportunistic routing protocols for wireless mesh networks. Opportunistic routing differs from traditional routing in that it exploits the broadcast nature of wireless medium and defers route selection after packet transmissions.

\section{System Model}

We consider the network modeled by a network graph $\mathrm{G}=(\mathrm{V}, \mathrm{E})$, where $\mathrm{V}$ represents the set of nodes in the network and $\mathrm{E}$ represents the set of bi-directional wireless links between the nodes. Each node $\mathrm{v}_{\mathrm{i}}$ is characterized by a circular transmission range $\mathrm{R}_{\mathrm{i}}$ and a carrier sensing range $R_{i}^{\prime}$ and equipped with multiple radio interfaces for communication with other nodes in the mesh network. We denote the neighborhood of node $\mathrm{v}_{\mathrm{i}}$ by $\mathrm{N}\left(\mathrm{v}_{\mathrm{i}}\right)$, consisting of nodes residing within its transmission range. We assume that each mesh node $\mathrm{v}_{\mathrm{i}}$ is equipped with $\mathrm{I}_{\mathrm{i}}>$ 1 radio interfaces, and there are $\mathrm{C}$ available channels in the network, where $1<=\mathrm{I}_{\mathrm{i}}<=\mathrm{C}$. To operate the radio interfaces simultaneously, they need to be tuned to orthogonal channels. We assume that $\mathrm{C}=\left\{\mathrm{c}_{1}, \mathrm{c}_{2}, \ldots \ldots ., \mathrm{c}_{\mathrm{C}}\right\}$ is the set of orthogonal channels available in the network and also that these channels are homogeneous in terms of the channel capacity. The number of available orthogonal channels depends on the radio frequency spectrum used. IEEE $802.11 \mathrm{~b}$ and IEEE 802.11a support 3 and 12 orthogonal channels, respectively. A pair of nodes $\mathrm{v}_{\mathrm{i}}$ and $\mathrm{v}_{\mathrm{j}}$, has a link $l_{i j}^{k}$, if they are physically located within each other's communication range and operating over channel $c_{k}$.

\subsection{Link Interference Domain}

Due to the sharing nature of wireless links, two nodes within the interference range of each other as to refrain themselves from simultaneous transmission on the same channel at the same time. We use the notion of interference domain to spot those contending links. The interference domain of a link $l_{i j}^{k}$ consists of set of links that interfere with $l_{i j}^{k}$ if they operate on the same channel as $l_{i j}^{k}$. We focus on the protocol model (Jain \& Padhye, 2005) in this paper to build a multi radio conflict graph (MCG) (Ramachandran \& Almeroth, 2006) to compute the interference domain, which is widely used for modeling interference in the wireless network. In this model, the interference between nodes is determined by the interference range, which is typically longer than 1 - hop communication range $\left(R_{i}^{\prime} \geq R_{i}\right)$. Accordingly, a transmission from node $\mathrm{v}_{\mathrm{i}}$ to node $\mathrm{v}_{\mathrm{j}}$ is successful provided no other node located within a distance $R_{i}^{\prime}$ from $v_{i}$ and $v_{\mathrm{j}}$ transmits at the same time. Formally, the interference domain of link $1_{\mathrm{ij}}$ operating over channel $c_{k}, I D_{i j}^{k}$ can be defined as follows:

$$
I D_{i j}^{k}=\left\{l_{u v}^{k} \in E \mid d_{i u}<R_{i}^{\prime} \vee d_{i v}<R_{i}^{\prime} \vee d_{j u}<R_{j}^{\prime} \vee d_{j v}<R_{j}^{\prime} \vee d_{u i}<R_{u}^{\prime} \vee d_{u j}<R_{u}^{\prime} \vee d_{v i}<R_{v}^{\prime} \vee d_{v j}<R_{v}^{\prime}\right\}
$$

where $d_{i u}$ is the Euclidian distance between node $\mathrm{v}_{\mathrm{i}}$ and $\mathrm{v}_{\mathrm{u}}$. Thus no other links in the interference domain of link $l_{i j}^{k}$ can be engaged in communication concurrently with link $l_{i j}^{k}$.

\section{Proposed EEC Metric}

This section introduces our new routing metric, called EEC that takes into consideration the number of interferer links (by capturing the complex interplay between the inter-flow and intra-flow interference), dynamic traffic load on links and packet delay.

\subsection{Problem Description and Motivation}

To select a routing path in WMNs, the routing algorithm needs to consider possible unreliable network topology due to the multihop wireless environment. In addition, the routing path selection is intertwined with resource allocation, interference avoidance and rate adaptation across multiple hops. Mobility in WMNs is less challenging than in MANETs, which is an advantage for designing protocols for WMNs and makes the 
performance of a routing protocol tractable in a multihop wireless mesh environment. The ultimate goal of a routing protocol is to find a routing path for any source-destination pair but also to achieve the best performance. The performance parameters are diverse and can be defined at different levels of the networking systems.

\subsubsection{Problems with Other Routing Metric}

In order to motivate the need for a new routing metric, in the following, we give an overview of the various routing metrics proposed for multi-hop wireless mesh networks in the literature and discuss their limitations.

\subsubsection{Hop Count}

Routing protocols, like AODV(Perkins \& Royer, 1999), DSR[(Johnson \& Maltz, 1996), DSDV(Perkins \& Bhagwat, 1994), forward traffic flows towards destination based on minimum hop count where packets are likely to track the same route leading to more interference and congestion and thus severe devastated throughput.

\subsubsection{Link Quality Based Routing Metric}

To incorporate the quality and data rate of forwarding links, quality-aware routing metrics have been proposed to improve the throughput of WMNs. For instance, ETX(Cout \& Aguayo, 2003) estimates average number of transmissions necessary to transfer a packet successfully over a wireless link. The path metric computed as the summation of ETX of each link in the path. Though ETX improves performance over minimum hop-count metric, it is unable to address inter-flow interference of multiple flows in the network. Here, the varying transmission rates of different wireless links and sizes of data packets are also ignored. ETX was originally designed for SRSC WMN, so in MRMC networks in the presence of multiple channels ETX is not capable of finding better paths exploiting channel diversity.

\subsubsection{Computing End-to-End Delay}

Routing protocols based on computing minimum end-to-end delay has been studied in (Draves \& Padhye, 2004), (Kim \& Jakllari, 2011) and (Islam \& Alam, 2010). In WCETT(Draves \& Padhye, 2004) performance of bottleneck channels are considered to compute the overall expected transmission time of the routing path. However, WCETT ignores the quality of transmitting link and number of flows travelling the contending link. Thus WCETT explicitly neglects the adverse effect of inter-flow interference. Therefore, when network is overwhelmed with flows, WCETT may lead to forwarding traffic through more congested area leading to degraded overall network throughput.

EFT (Islam \& Alam, 2010) estimates end-to-end forwarding time of a packet on a multi hop path by measuring packet transmission delay, queuing delay and idle slots due to contending nodes at each forwarding node. A node situated in congested vicinity is bound to experience much interruption due to contending nodes' flow rates, traffic loads and priorities. EFT parameter is unable to capture intra flow interference. EED metric proposed in ( $\mathrm{Li}, \&$ Cheng, 2013)is the average delay for a packet over a link consisting of the queuing delay and the transmission delay considering link quality, MAC collision, and hop count. The EED metric is combined with MRAB metric to form a weighted end-to-end delay (WEED) metric, where MRAB describes the impact due to intra-flow and inter-flow interference in the MR-MC context.

\subsubsection{Computing Interference}

In a dense network, selection of a route with higher bandwidth and less error rate between every pair of communicating nodes may result in severe interference that may deteriorate the quality of the remaining available resources. As a consequence, new arriving flows will experience poor services. The philosophy behind (Langar \& Bouabdallah, 2009) and (Subramanian \& Buddhikot, 2006)is then to choose a good path that alleviates the resulting interference in order to preserve good paths for the newly arriving flows. As a result, the total network throughput will be maximized. The INX (Langar \& Bouabdallah, 2009) of a link is defined as the product of the ETX of the link and the bit rate of each interferer link resulting from a transmission on that link. Clearly, The INX metric captures the quality of the wireless link by including the ETX (Cout \& Aguayo, 2003) metric and the volume of neighboring interference by incorporating interfering links traffic load. The interference ratio parameter proposed in iAWARE (Subramanian \& Buddhikot, 2006) computes the interference level of a link of a node by computing the ratio between SINR and SNR value experienced at the node. This parameter is then incorporated with ETT (Draves \& Padhye, 2004) to find the forwarding time of a packet. When there is no interference in the network, ETT captures the quality of the link quite well as links with less expected transmission time give better throughput. But when there are more interfering flows in the network, the varying interference experienced by a link is captured by interference ratio parameter and factored into the routing metric to find good quality paths. 
Considering the shortcomings of above discussed proposals, we aimed to design a routing metric that utilizes the enhanced capacity of MRMC WMN by computing achievable link bandwidth which is influenced by degree of interfering neighboring channels, traffic volume and packet transmission delay of forwarding links. The computed route offers the maximum possible bandwidth towards destination while curtailing interference, alleviating congestion and balancing load throughout the network.

\subsection{EEC}

In order to transfer a packet, a source node computes set of links towards the destination that offers highest throughput, minimum delay and guaranteed delivery. A forwarding link is characterized by its quality, capacity, traffic demand and the degree of intervention experienced due to inter-flow and intra-flow interference. Thus the bandwidth actually attainable on a link for a flow is affected by those link properties. From the points discussed above, we put endeavor to form a routing metric that aims to compute the attainable bandwidth for a flow over a path which actually reflects congestion, node delay and traffic pressure on the desired path.

\subsubsection{Intra Flow Interference}

The capacity of each link $l_{i j}^{k}$ is considered to be identical. The effect of the interference is to prevent simultaneous transmissions over neighboring links using the same channel. Hence, the throughput that can be achieved across a wireless link (denoted as allotted bandwidth) is affected by the number of interfering neighboring links. To find the allotted bandwidth of link $l_{i j}^{k}$, we need to find the other links that interfere with $\operatorname{link} l_{i j}^{k}$. Intra flow interference cost, represented by $\cos t_{-} \operatorname{In} f_{i j}^{k}$, is represented by the number of links contained in the Interference Domain of link $l_{i j}^{k}$, computer as in Eq. (1).

$$
\cos t_{-} \inf _{i j}^{k}=\left|I D_{i j}^{k}\right|
$$

If the bandwidth of the link $l_{i j}^{k}$ is $B_{i j}^{k}$, then to find the allotted bandwidth, $B_{-} a l t_{i j}^{k}$ of link $l_{i j}$ under intra-flow interference, we use Eq. (2).

$$
B_{-} a l t_{i j}^{k}=\frac{B_{i j}^{k}}{\cos t_{-} \inf _{i j}^{k}}
$$

\subsubsection{Inter Flow Interference and Traffic Load}

In our proposed work, each dynamic interface in each node is responsible for forwarding data to multiple downstream nodes. So each interface maintains one designated queue for each forwarding flow. The data to be sent to each downstream are buffered in the corresponding queue. In order to precisely rectify current load level of a link $l_{i j}^{k}$; node $\mathrm{v}_{\mathrm{i}}$ uses following parameters: i) queue utilization level for each downstream link $l_{i j}^{k}$, $Q_{-} U t l_{i j}^{k}$ and ii) congestion degree for each downstream link $l_{i j}^{k}, C D_{i j}^{k}$. Suppose the current queue size at node $\mathrm{v}_{\mathrm{i}}$ for $l_{i j}^{k}$ is $Q_{-}$curr $_{i j}^{k}$ then the average queue size $Q_{-} a v g_{i j}^{k}$, is computed using EWMA (exponential weighted moving average) algorithm as follows in Eq. (3):

$$
Q_{-} a v g_{i j}^{k}=(1-\beta) \times Q_{-} a v g_{i j}^{k}+\beta \times Q_{-} c u r r_{i j}^{k}
$$

where $\beta$ is a weight factor. A value of $\beta$ closer to 1 , calculates the average queue size giving more emphasis on the current queue size $Q_{-} C u r r_{i j}^{k}$. The queue utilization ratio for link $l_{i j}^{k}$, is computed as Eq. (4):

$$
Q_{-} U t l_{i j}^{k}=\frac{Q_{-} a v g_{i j}^{k}}{Q_{-} \max _{i j}^{k}}
$$

where $Q_{-} \max _{i j}^{k}$ is the maximum length of a queue. The congestion degree $C D_{i j}^{k}$ is proposed to reflect the current congestion intensity at each $\operatorname{link} l_{i j}^{k} . C D_{i j}^{k}$ is defined as the ratio of the mean packet service time $T_{-} s r v_{i j}^{k}$ over the mean packet inter-arrival time $T_{-} a r r_{i j}^{k}$ over a predefined time interval in node $\mathrm{v}_{\mathrm{i}}$ as in Eq. 
(5).

$$
C D_{i j}^{k}=\frac{T_{-} s e r v_{i j}^{k}}{T_{-} a r r_{i j}^{k}}
$$

Here the mean packer inter-arrival time $T_{-} a r r_{i j}^{k}$, is defined as the interval between the arrival of two adjacent data packets from upstream traffic, and the mean packet service time $T_{-} s r v_{i j}^{k}$, is defined as the average processing time of the data packets over link $l_{i j}^{k}$ at node $\mathrm{v}_{\mathrm{i}}$.

To calculate the time taken for a packet to be delivered over a link, the following four components are needed to be measured: (i) the transmission time $\left(T_{-} t r_{i j}^{k}\right)$ of a packet is the duration wherein the packet uses the wireless medium, (ii) the backoff delay $T_{-} b c k_{i j}^{k}$ is the duration of the backoff slots that a node requires to access the wireless medium. (iii) The freezing time $T_{-} f r z_{i j}^{k}$ represents (1) the number of interruptions during backoff process period, which is significant if of the number of contending nodes and their loads is high, (2) the duration of the interruption that captures the flow rate and the size of the contending nodes, and (iv) the queuing delay $T_{-} q_{i j}^{k}$ of a packet is the difference between times when the packet enters the queue and the packet starts the first transmission attempt.

Thus packet service time $T_{-} s r v_{i j}^{k}$ is calculated as in Eq. (6), considering that a successful link layer transmission requires $n$ number of retransmission.

$$
T_{-} s r v_{i j}^{k}=T_{-} q_{i j}^{k}+T_{-} b c k_{i j}^{k}(n)+T_{-} f r z_{i j}^{k}(n)+n \times T_{-} t r_{i j}^{k}
$$

The computation of delay parameters are described below:

- $\quad T_{-} t r_{i j}^{k}$ calculation: The transmission time depends on the channel capacity and the size of the packet. If the size of the packet is $\mathrm{S}$ and data rate is B then $T_{-} t r_{i j}^{k}$ is calculated as in Eq. (7)

$$
T_{-} t r_{i j}^{k}=\frac{S}{B}
$$

$T \_b c k_{i j}^{k}$ calculation: At the beginning of the backoff process of the $m^{\text {th }}$ transmission attempt, the 802.11 MAC randomly selects the back-off window $C W_{m}$ from the interval $\left(0,2^{m-1} C W_{\min }\right)$, where $C W_{\min }$ is the minimum back-off window size. The interval increases to $\left(0, C W_{\max }\right)$ with the attempts, and after that, there is no longer an increase; $C W_{\max }$ is the maximum back-off window size. We assume that $C W_{\max }=2^{6} C W_{\min }$ conforming to the IEEE 802.11 standard (IEEE Standard for MAC, 2006). Then, as derived in (Draves \& Padhye, 2004) we can express $T_{-} b c k_{i j}^{k}$ as Eq.(8).

$$
\begin{aligned}
& T_{-} b c k_{i j}^{k}(n)=T_{\text {slot }} \sum_{m=1}^{n} E\left[C W_{m}\right]
\end{aligned}
$$

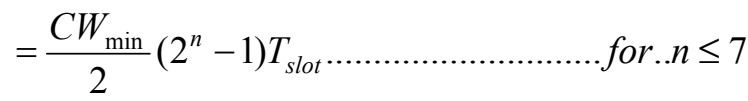

$$
\begin{aligned}
& =\frac{C W_{\min }}{2}(63+64(n-7)) T_{\text {slot }} \ldots \ldots \ldots \ldots \ldots . . . \text { for } . . n \geq 8
\end{aligned}
$$

where $E\left[C W_{m}\right]$ denotes the expected value of $C W_{m}$ and $T_{\text {slot }}$ denotes the size of each slot in time units.

$T_{-} f_{i j}^{k}$ calculation: The calculation of backoff delay of link $l_{i j}$ captures the interfering neighborhood density and traffic demand, but ignores volume of traffic transmitted and channel condition experienced by contending links. During the backoff procedure of link $l_{i j}$, if any other link belonging to the $I D_{i j}^{k}$ detains the medium, then link $l_{i j}$ has to freeze its backoff counter until the medium is free. The freezing time is greatly affected by the volume of the traffic transferred and the quality of the link (as the link quality deteriorates the data rate of the channel). Thus, during $m^{\text {th }}$ backoff interval, freezing time experienced by link $l_{i j}$ is calculated as in Eq. (9).

$$
T_{-} f_{i j}^{k}=E\left[\operatorname{Int}_{i j}\right] \times E\left[\operatorname{Int}_{-} d_{i j}\right]
$$

where $E\left[\operatorname{Int}_{i j}\right\rfloor$ is the expected number of interruption during $m^{\text {th }}$ transmission attempt and $E\left[\right.$ Int $\left.\_d_{i j}\right]$ is the duration of the interruption. Each node measures $E\left[\operatorname{Int} t_{i j}\right]$ and $E\left[\operatorname{Int} t_{-} d_{i j}\right]$ using exponentially weighted moving average (EWMA). 
$T_{-} q_{i j}^{k}$ calculation: Each packet is time-stamped when it is enqueued and when it is to be transmitted. The difference between the two time-stamps represents the queuing delay of the packet. Let $Q_{-} d e l_{i j}^{t}$ be the measured queuing delay of the $t^{\text {th }}$ enqueued packet at the transmitter of a link $l_{i j}$. The average queuing delay $T_{-} q_{i j}^{k}$ for link $l_{i j}$ is estimated by using the following exponential smoothing formula given in Eq. (10):

$$
T_{-} q_{i j}^{k}=\alpha \times Q_{-} d e l_{i j}^{t}+(1-\alpha) \times A v g_{-} Q_{-} d e l_{i j}
$$

where $\alpha$ is the smoothing factor $(0<\alpha<1)$. $A v g_{-} Q_{-} d e l_{i j}^{t}$ is the mean of the queuing delays of the last $k$ enqueued packets. If $\alpha \approx 1$ there is a lesser extent of smoothing and recent changes are considered more important. In our experiments, we choose $\alpha=0.5$ to balance the two factors.

Finally, the link load level $\operatorname{Load}_{i j}^{k}$ of link $l_{i j}$ is calculated as given in Eq.(11).

$$
\operatorname{Load}_{i j}^{k}=Q_{-} U t t_{i j}^{k} \times C D_{i j}^{k}
$$

\subsubsection{EEC Computation}

Given the allotted bandwidth of the link $l_{i j}$ to find the expected effective capacity, $E E C_{i j}^{k}$, of link $l_{i j}$ under interflow and intraflow interference, we use Eq. (12).

$$
E E C_{i j}^{k}=\left(1-\operatorname{Load}_{i j}^{k}\right) \times B_{-} a l t_{i j}^{k}
$$

Thus the EEC along a $\mathrm{H}$ hop path is computed as in Eq. (13).

$$
E E C=\min _{l_{i j}}\left(E E C_{i j}^{k}\right)
$$

\section{Performance Evaluation}

To assess the performance of our proposed scheme, we have performed extensive simulation using ns-2 simulator (ns-2, 2008).

\subsection{Simulation Metric and Parameters}

The simulation parameters are set as follows. 100 nodes are randomly distributed in a square region of $1000 \mathrm{~m} \times$ $1000 \mathrm{~m}$. Each node has two radios and each radio operates on one of the three orthogonal channels available in $802.11 \mathrm{~b}$. For the simulation setup, the transmission range and carrier sense range are set to $250 \mathrm{~m}$ and $550 \mathrm{~m}$, respectively. The size of each packet is 512 Bytes. We generate UDP packets for all flows at a constant rate and pick sources and destinations randomly. The routing protocol used is AODV (Adhoc on Demand Distance Vector Routing). The buffer size at each node is 100 packets.

The following metrics has been realized to validate the efficiency of our proposed scheme. (i) Throughput, (ii) Delivery ratio and (iii) End-to-end delay.

\subsection{Simulation Results and Discussion}

Simulation results are obtained under several different scenarios to demonstrate the impact of network size, number of available orthogonal channels and traffic load on simulation parameters. Each scenario is given an initial channel allocation according to CLICA (Marina \& Samir, 2010) algorithm. In the proposed heuristic channel assignment model, priority is given to links closer to gateway and carrying heavy traffic load. A greedy approach finds the channel that will minimize the maximum network interference while preserving the network connectivity. The result of proposed work has been compared with ICAR (Ding \& Yong, 2013) and EFT (Islam $\&$ Alam, 2010). Each simulation is carried 100 times to obtain the average performance.

\subsubsection{Scenario 1: Varying Network Size}

In this scenario, we gradually increase the number of nodes in the network from 10 to 100 nodes and observed the performance behavior of the system. While increasing the number of nodes, we maintained the same node density and considered 20 flows transmitting at a rate of 20 packets/second (pps). The result of this scenario shown in Figures 1, 2 and 3.

In Figure 1, we can identify that with an increase in the number of mesh nodes, end-to-end delay increases as the number of hop distance between each source-destination pair become greater. ECC has been shown to achieve less average end-to-end delay as each link constituting the path towards destination in EEC has less number of conflicting links, less waiting packets at link buffers and minimum packet service time. ICAR deduces capacity 
of each link using ETX of that link scaled down according to the level of traffic pressure on its interfering links. EFT is able to minimize delay over ICAR, as it computes node delay taking into consideration queuing time, packet transmission time and traffic load on interfering neighborhood.

In Figure 2, the throughput has an inverse relationship with the number of nodes due to high loss of data packets. As shown in Figure 2, ECC is able to retain higher average network throughput with an increasing number of nodes as compared to other routing metrics. ECC metric captivates the actual capacity of a link that guarantees a high throughput path towards destination around less-congested areas.

Figure 3 shows the trend in delivery success with increasing nodes. The loss rates are low for a network with small number of nodes because of lower interferences and number of hops among the nodes. The loss rate increases with the network size. EEC outperforms the other schemes significantly.

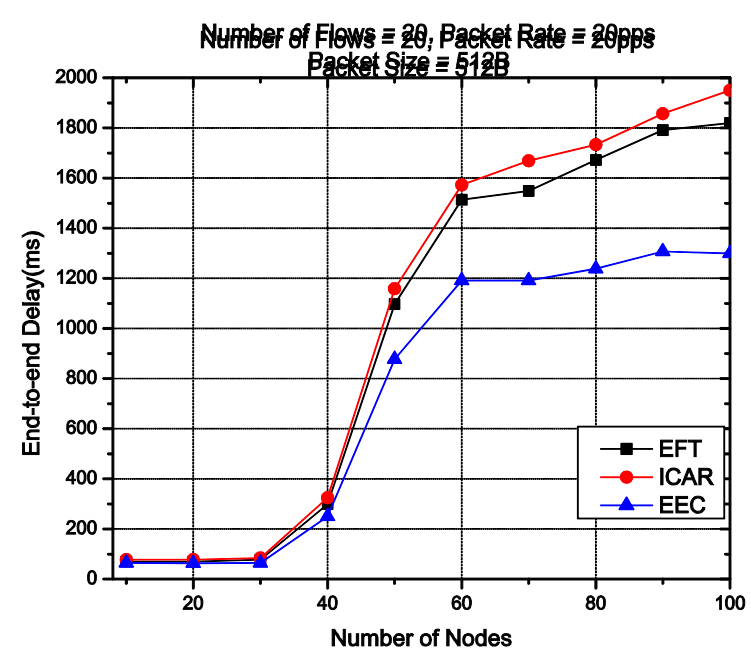

Figure 1. Number of nodes vs end-to-end delay

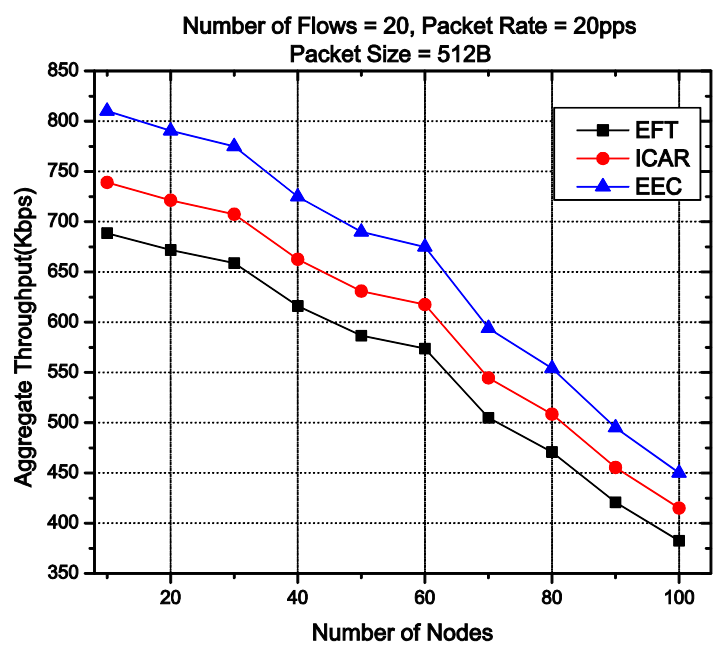

Figure 2. Number of nodes vs throughput 


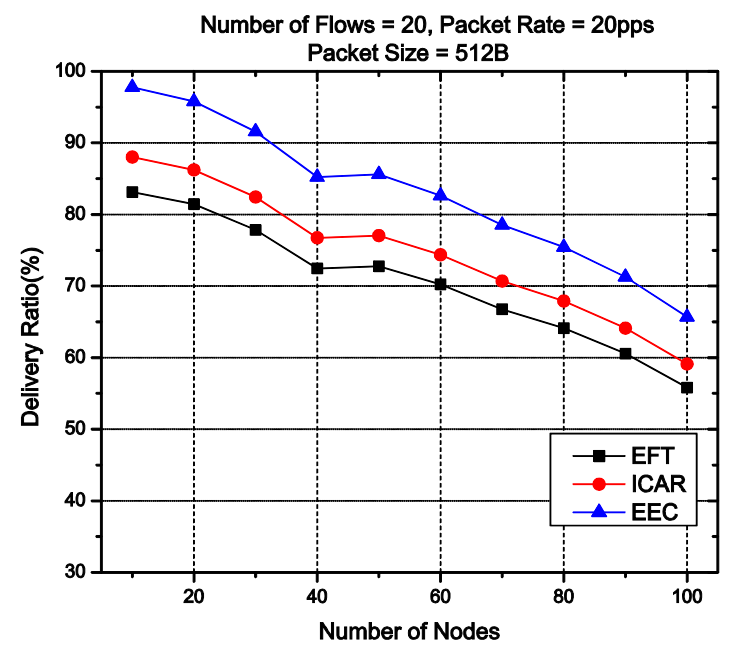

Figure 3. Number of nodes vs packet delivery ratio

\subsubsection{Scenario 2: Varying Traffic Load}

Here, we vary transmission rate to study the impact of traffic load. The packet sending rate varies from 5 to $100 \mathrm{pps}$, while the number of flows from sources to destination is 20 and the number node is 100 (as shown in Figures 4, 5 and 6).

In Figure 4, transmission rate has direct relationships with end to end delay. With the increased traffic load, the neighboring nodes experience higher contention, resulting in increased back-off time at MAC layer. It may increase the waiting time of packets in a queue, cause an overflow queue problem and may prevent new arrival of packets. As a result new packet will be lost, and retransmission mechanism will take place. This process will continue and may cause very high delay. Our proposed work has significantly achieved reduction of end to end delay over the EFT and ICAR.

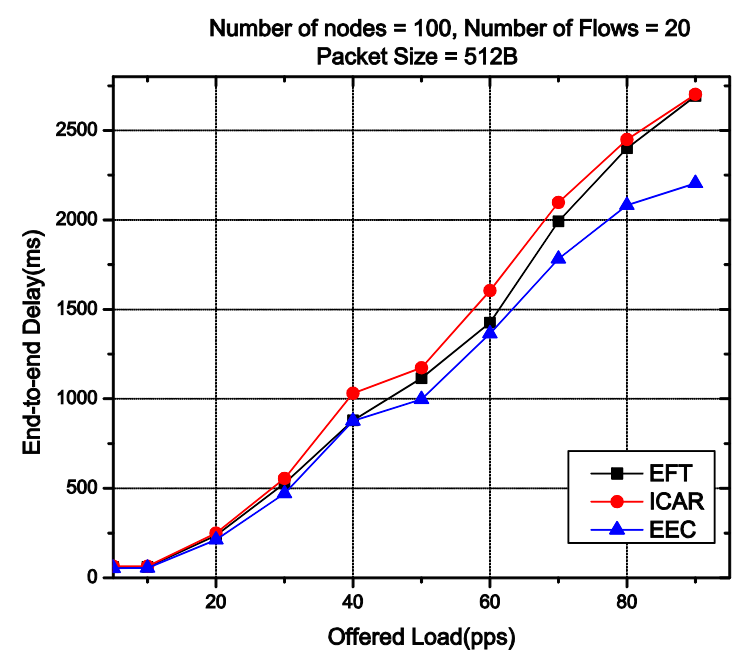

Figure 4. Offered load vs. end-to-end delay

Figure 5 shows the aggregated throughput versus the traffic demand per flow. ICAR selects the path based on the link quality and data rate of forwarding node and traffic load of contending neighbors. The number of contending nodes and their loads effects the path selection in EFT. Proposed ECC achieves higher throughput by selecting the path that offers highest capacity in terms of least interference, higher bandwidth and minimum packet forwarding time. 


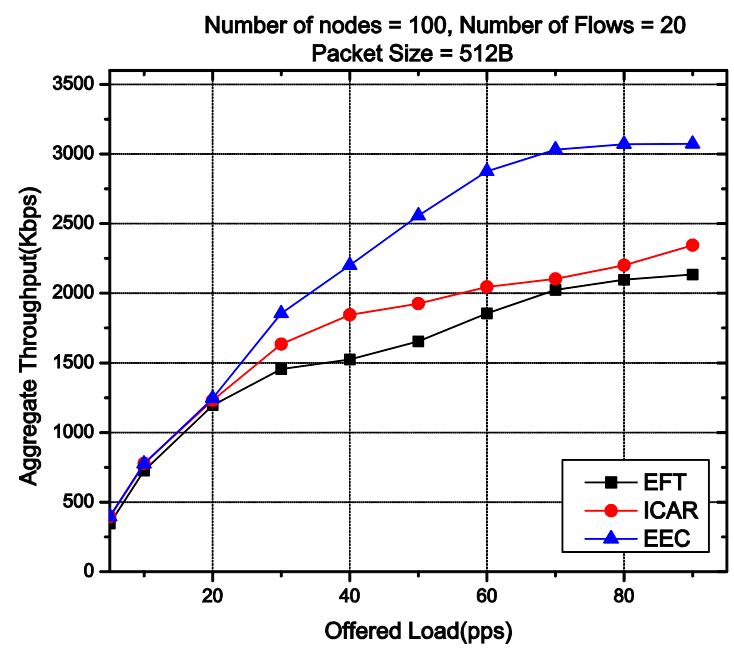

Figure 5. Offered load vs. throughput

Figure 6 shows the packet delivery ratio of the network with increasing traffic loads. ECC routes packets through less congested regions of the network. Therefore, as the packets experience less contention, loss rate in ECC is lower than other metrics.

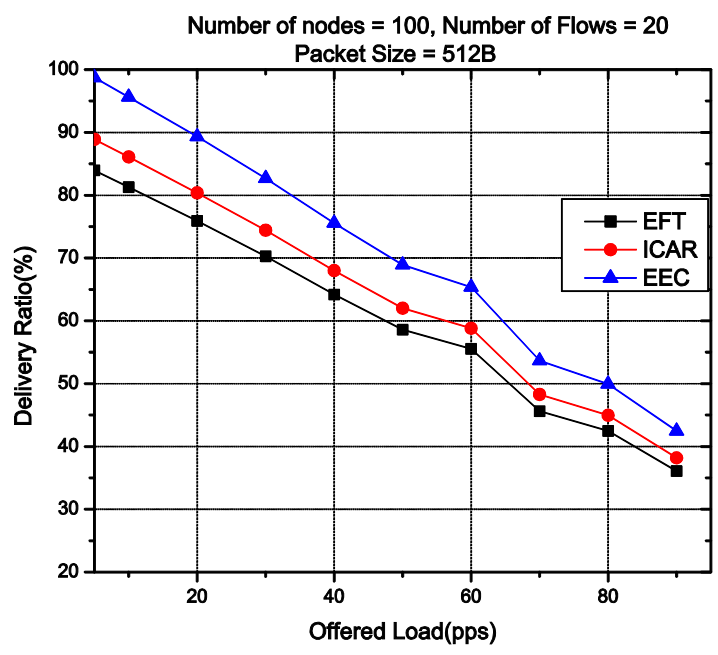

Figure 6. Offered Load vs. packet delivery ratio

\subsubsection{Scenario 3: Varying Number of Flows}

To study the impact of increasing number of traffic flows in a network, we conducted simulation on a 100 node network where the injection of flows varied from 10 to 100 considering random source and destination pair while each flow having same packet transmission rate.

Figure 7 compares the end-to-end packet delay under different routing metrics with increasing traffic flows. Increased number of flows causes increased packet forwarding time, buffer overflow and retransmission. Thus end-to-end delay rises significantly with number of flows. Our proposed EEC metric attains overall reduced end-to-end delay over EFT and ICAR as it routes data through least congested links that exhibit low buffer occupancy, least contention and forwarding time. 


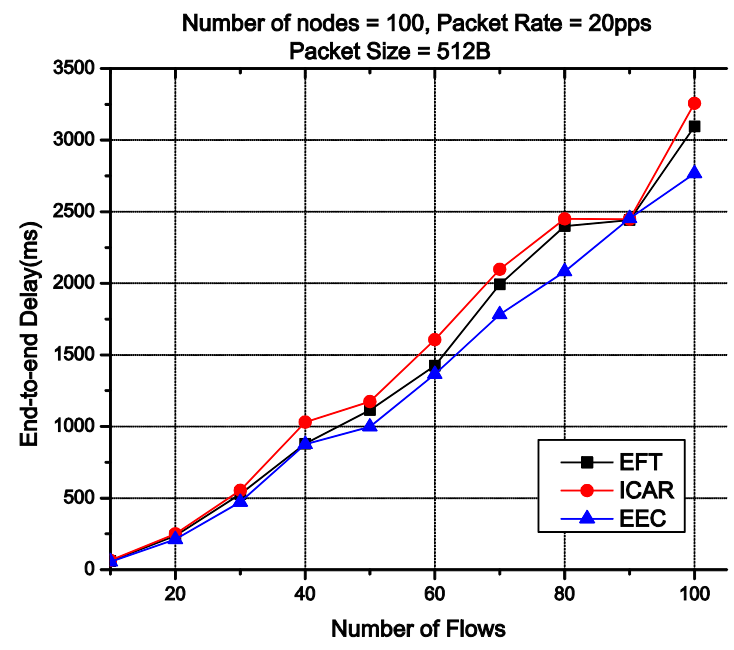

Figure 7. Traffic flows vs. end-to-end packet delay

Throughput comparison under different routing metric with varying traffic flows has been shown in Figure 8 . EFT computes packet forwarding time along a path that incorporates inter-flow interference but unable to address intra-flow interference. As a consequence with increasing number of flows, the selected target path experience exhaustive interference, resulting in overall network throughput degradation. ICAR computes attainable bandwidth over a link considering link's data rate, load and ETX; and set of interferer links. To estimate the load status of a link only the queue length is monitored which does not reflect the actual congestion status of a link. As a consequence with increasing flow demand, paths are chosen that lead to more congestion, resulting in more packet drops and network experience reduced throughput. Our proposed EEC metric captures inter-flow interference, intra-flow interference and current link load to select a path that necessarily avoids densely loaded areas and thus balances load over the network which significantly improves system throughput.

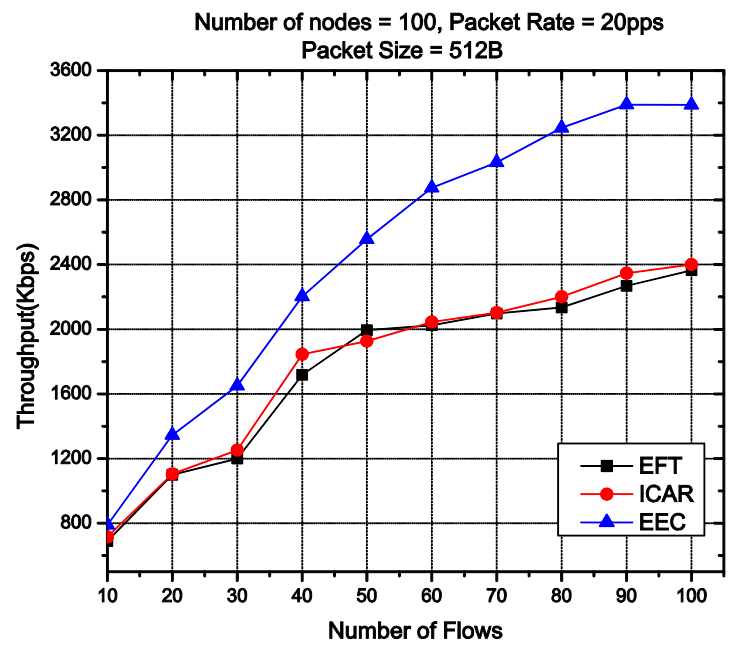

Figure 8. Traffic flows vs. throughput

Use of our proposed metric EEC has shown to achieve better packet delivery rate compared to other routing metric which is revealed in Figure 9. 


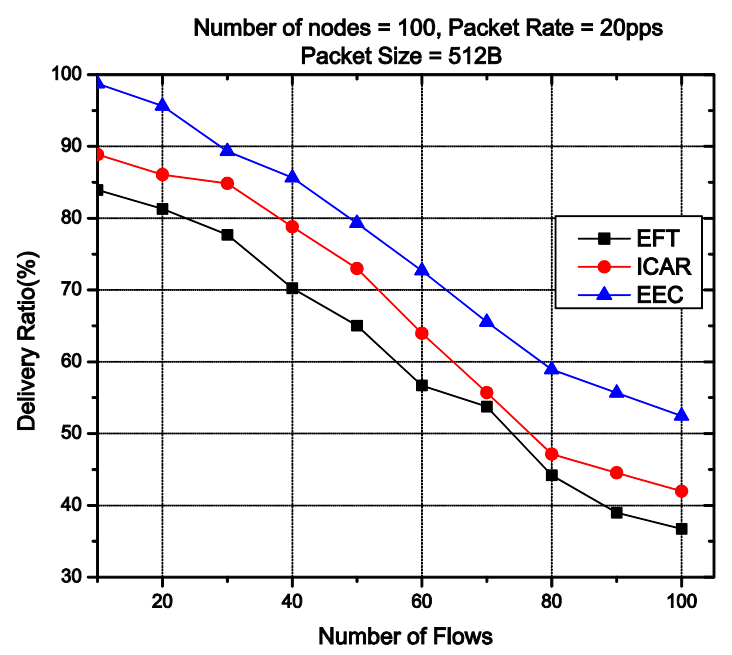

Figure 9. Traffic flows vs. delivery ratio

\section{Conclusion and Future Work}

In this paper, we proposed a novel interference-aware and traffic-load sensitive routing metric for WMN. Our target was to maintain higher network throughput while avoiding congestion, mitigate interference and balancing network load. The significant contributions to this paper are: (i) calculating link allotted bandwidth due to interflow interference in a distributed manner, (ii) the use of elementary in-node parameters: packet buffer occupancy, queuing delay, backoff time, freezing time and packet transmission delay to compute link load and (iii) combining (i) and (ii) to formulate the routing metric EEC. Through ns-2 simulations, we found that the employment of our proposed routing metric avoids congested route and greatly reduces the neighboring hop interference, which in turn increases delivery ratio and network throughput.

In order to utilize the increased capacity of a MRMC WMN routing problem should be jointly studied with channel assignment problem. Our future plan is to incorporate channel reallocation in top of our proposed EEC routing metric to reduce neighborhood link interference, improve offered bandwidth, balance network load and thus amplify network throughput.

Our proposed routing protocol works in a distributed manner which incurs exchange of lots of control message throughout the network consuming more bandwidth. Our future study will reveal the impact of distributed approach and thus propose a mechanism that may help nodes to compute route and channel reallocation in an effective manner that may reduce computational overhead and transmission of control message.

\section{References}

Akyildiz, I. F., Wang, X., \& Wang, W. (2005). Wireless mesh networks: A survey. Computer networks, 47(4), 445-487. http://dx.doi.org/10.1016/j.comnet.2004.12.001

Alicherry, M., Bhatia, R., \& Li, L. E. (2006). Joint channel assignment and routing for throughput optimization in multiradio wireless mesh networks. IEEE Journal on Selected Areas in Communications, 24(11), 1960-1971. http://dx.doi.org/10.1109/JSAC.2006.881641

Biswas, S., \& Morris, R. (2005, August). ExOR: opportunistic multi-hop routing for wireless networks. In ACM SIGCOMM Computer Communication Review (Vol. 35, No. 4, pp. 133-144). ACM. http://dx.doi.org/10.1145/1090191.1080108

De Couto, D. S., Aguayo, D., Bicket, J., \& Morris, R. (2005). A high-throughput path metric for multi-hop wireless routing. Wireless Networks, 11(4), 419-434. http://dx.doi.org/10.1007/s11276-005-1766-z

Dhananjay, A., Zhang, H., Li, J., \& Subramanian, L. (2009, August). Practical, distributed channel assignment and routing in dual-radio mesh networks. InACM SIGCOMM Computer Communication Review (Vol. 39, No. 4, pp. 99-110). ACM. http://dx.doi.org/10.1145/1594977.1592581

Ding, Y., Pongaliur, K., \& Xiao, L. (2013). Channel allocation and routing in hybrid multichannel multiradio wireless mesh networks. Mobile Computing, IEEE Transactions on, 12(2), 206-218. 
http://dx.doi.org/10.1109/TMC.2011.261

Draves, R., Padhye, J., \& Zill, B. (2004, September). Routing in multi-radio, multi-hop wireless mesh networks. In Proceedings of the 10th annual international conference on Mobile computing and networking (pp. 114-128). ACM.

Gálvez, J. J., \& Ruiz, P. M. (2013). Efficient rate allocation, routing and channel assignment in wireless mesh networks supporting dynamic traffic flows. Ad Hoc Networks, 11(6), 1765-1781. http://dx.doi.org/10.1016/j.adhoc.2013.04.002

Gardellin, V., Das, S. K., Lenzini, L., Cicconetti, C., \& Mingozzi, E. (2011). G-PaMeLA: A divide-and-conquer approach for joint channel assignment and routing in multi-radio multi-channel wireless mesh networks. Journal of parallel and distributed computing, 71(3), 381-396. http://dx.doi.org/10.1016/j.jpdc.2010.10.008

Hong, W. W., Long, F., Xia, P., \& Chan, S. H. G. (2012, July). Distributed Joint Channel and Routing Assignment for Multimedia Wireless Mesh Networks. InMultimedia and Expo (ICME), 2012 IEEE International Conference on (pp. 404-409). IEEE.

IEEE 802.11s Task Group. (2008). Draft amendment to standard for information technology-telecommunications and information exchange between systems-local and metropolitan area networks-specific requirements - part 11: wireless lan medium access control (MAC) and physical layer (PHY) specifications: amendment IEEE p802.11s/d2.02: mesh networking.

IEEE Standard for Wireless LAN Medium Access Control (MAC) and Physical Layer (PHY) specications. ISO/IEC 8802-11:2005/Amd.4:2006(E).

Information Sciences Institute (2008). ns-2 network simulator. Software package. Retrieved from $\mathrm{http}: / /$ www.isi.edu/nsnam/ns/

Islam, M. S., Alam, M. M., Hamid, M. A., Hong, C. S., \& Lee, S. (2010). EFT: a high throughput routing metric for IEEE $802.11 \mathrm{~s}$ wireless mesh networks. annals of telecommunications-annales des télécommunications, 65(5-6), 247-262. http://dx.doi.org/10.1007/s12243-009-0130-1

Jain, K., Padhye, J., Padmanabhan, V. N., \& Qiu, L. (2005). Impact of interference on multi-hop wireless network performance. Wireless networks, 11(4), 471-487. http://dx.doi.org/10.1007/s11276-005-1769-9

Jakllari, G., Eidenbenz, S., Hengartner, N., Krishnamurthy, S. V., \& Faloutsos, M. (2012). Link positions matter: A noncommutative routing metric for wireless mesh networks. Mobile Computing, IEEE Transactions on, 11(1), 61-72. http://dx.doi.org/10.1109/TMC.2011.79

Johnson, D. B., \& Maltz, D. A. (1996). Dynamic source routing in ad hoc wireless networks. In Mobile computing (pp. 153-181). Springer US. http://dx.doi.org/10.1007/978-0-585-29603-6_5

Kim, T. S., Jakllari, G., Krishnamurthy, S. V., \& Faloutsos, M. (2011, October). A Unified Metric for Routing and Rate Adaptation in Multi-rate Wireless Mesh Networks. In Mobile Adhoc and Sensor Systems (MASS), 2011 IEEE 8th International Conference on (pp. 242-251). IEEE.

Langar, R., Bouabdallah, N., \& Boutaba, R. (2009). Mobility-aware clustering algorithms with interference constraints in wireless mesh networks. Computer Networks, 53(1), 25-44. http://dx.doi.org/10.1016/j.comnet.2008.09.012

Li, H., Cheng, Y., \& Zhou, C. (2008, November). Multi-hop effective bandwidth based routing in multi-radio wireless mesh networks. In Global Telecommunications Conference, 2008. IEEE GLOBECOM 2008. IEEE (pp. 1-5). IEEE.

Li, H., Cheng, Y., Zhou, C., \& Zhuang, W. (2013). Routing Metrics for Minimizing End-to-End Delay in Multiradio Multichannel Wireless Networks. Parallel and Distributed Systems, IEEE Transactions on, 24(11), 2293-2303. http://dx.doi.org/10.1109/TPDS.2012.327

Li, X., Wu, J., Lin, S., \& Du, X. (2012). Channel switching control policy for wireless mesh networks. Journal of Parallel and Distributed Computing, 72(10), 1295-1305. http://dx.doi.org/10.1016/j.jpdc.2012.06.008

Marina, M. K., Das, S. R., \& Subramanian, A. P. (2010). A topology control approach for utilizing multiple channels in multi-radio wireless mesh networks. Computer networks, 54(2), 241-256. http://dx.doi.org/10.1016/j.comnet.2009.05.015

Mohsenian-Rad, A. H., \& Wong, V. W. (2007). Joint logical topology design, interface assignment, channel 
allocation, and routing for multi-channel wireless mesh networks. Wireless Communications. IEEE Transactions on, 6(12), 4432-4440. http://dx.doi.org/10.1109/TWC.2007.060312

Perkins, C. E., \& Bhagwat, P. (1994, October). Highly dynamic destination-sequenced distance-vector routing (DSDV) for mobile computers. In ACM SIGCOMM Computer Communication Review (Vol. 24, No. 4, pp. 234-244). ACM. http://dx.doi.org/10.1145/190809.190336

Perkins, C. E., \& Royer, E. M. (1999, February). Ad-hoc on-demand distance vector routing. In Mobile Computing Systems and Applications, 1999. Proceedings. WMCSA'99. Second IEEE Workshop on (pp. 90-100). IEEE.

Ramachandran, K. N., Belding-Royer, E. M., Almeroth, K. C., \& Buddhikot, M. M. (2006, April). Interference-Aware Channel Assignment in Multi-Radio Wireless Mesh Networks. In INFOCOM (Vol. 6, pp. 1-12).

Ramachandran, K., Sheriff, I., Belding, E. M., \& Almeroth, K. C. (2008). A multi-radio 802.11 mesh network $\begin{array}{llll}\text { architecture. Mobile Networks and 13(1-2), } & \text { 132-146. }\end{array}$ http://dx.doi.org/10.1007/s11036-008-0026-8

Rozner, E., Seshadri, J., Mehta, Y., \& Qiu, L. (2009). SOAR: Simple opportunistic adaptive routing protocol for wireless mesh networks. Mobile Computing, IEEE Transactions on, 8(12), 1622-1635. http://dx.doi.org/10.1109/TMC.2009.82

Subramanian, A. P., Buddhikot, M. M., \& Miller, S. (2006, September). Interference aware routing in multi-radio wireless mesh networks. In Wireless Mesh Networks, 2006. WiMesh 2006. 2nd IEEE Workshop on (pp. 55-63). IEEE.

Yang, Y., Wang, J., \& Kravets, R. (2005, September). Designing routing metrics for mesh networks. In IEEE Workshop on Wireless Mesh Networks (WiMesh).

Yuan, Y., Yang, H., Wong, S. H., Lu, S., \& Arbaugh, W. (2005, September). ROMER: resilient opportunistic mesh routing for wireless mesh networks. InIEEE workshop on wireless mesh networks (WiMesh) (Vol. 12).

\section{Copyrights}

Copyright for this article is retained by the author(s), with first publication rights granted to the journal.

This is an open-access article distributed under the terms and conditions of the Creative Commons Attribution license (http://creativecommons.org/licenses/by/3.0/). 